\title{
Kabuki Syndrome in a Newborn with a Complex Left- Sided Cardiac Lesion and Persistent Hypoglycemia due to Hyperinsulinism
}

Subhadra Ramanathan MS, MSc, Robin Dawn Clark MD

\section{Case History:}

A genetics consult was requested for a 6-week old term female infant for suspected Kabuki syndrome because of persistent hypoglycemia and presumed hyperinsulinism. This infant had Shone's Complex, severe hypoplastic transverse arch, and coarctation of the aorta. Her heart defect had been detected prenatally on fetal ultrasound. The pregnancy was complicated by polyhydramnios in the third trimester. The baby was born at term to a 31-year old G4P2112 mother with these growth parameters:

Birth weight: $2965 \mathrm{~g}$ (6 lb $8.6 \mathrm{oz})\left(21.97^{\text {th }}\right.$ percentile)

Birth length: $49.5 \mathrm{~cm}\left(19.49^{\prime \prime}\right)\left(39.05^{\text {th }}\right.$ percentile)

Birth head circumference: $34 \mathrm{~cm}$ (13.39") (50.01st percentile)

Her heart defect was the initial focus of medical attention, and she had cardiac surgery at one week of age: modified Norwood procedure, open atrial septectomy, right modified Blalock-Taussig shunt, and PDA ligation. Her postoperative course was complicated by two further aortic arch reconstruction procedures, plication of the right hemidiaphragm, and gastrostomy tube placement. Hypothyroidism was treated with Synthroid. Chromosome microarray analysis was normal.

\section{"Her postoperative course was complicated by two further aortic arch reconstruction procedures, plication of the right hemidiaphragm, and gastrostomy tube placement. Hypothyroidism was treated with Synthroid. Chromosome microarray analysis was normal."}

Although early glucose levels were stable at 50-70 until ten days of age, by postoperative day 2 , she had hypoglycemia (glucose 29 ), treated with D25 bolus $\times 1$. By one month of age, she had persistent hypoglycemia. She required progressively more glucose to stabilize her: first D10 bolus $x 5$, then continuous IV D15 and, subsequently, D30. She had been weaned to D25 at the time of the Genetics consultation. She had detectable levels of insulin during her hypoglycemic episodes, which, with a low betahydroxybutyrate level, suggested hyperinsulinism. Subsequently, she had a trial of Diazoxide, which was discontinued in favor of octreotide. Currently, at 12 weeks of age, her glucose levels are normal without treatment.

\section{Genetics Evaluation:}

Because of restrictions due to the coronavirus pandemic, this evaluation was done at a distance. The infant was in the cardiac intensive care unit at the time of consult. Video examination of the infant was facilitated by unit staff. The examination revealed a dysmorphic infant with microcephaly and hypotonia. She had long palpebral fissures that were everted laterally and faint eyebrows. She had prominent fetal pads on all fingertips.

Her clinical features, including the cardiac defect, hypoglycemia, facial features, and fetal finger pads, suggested Kabuki syndrome (KS) to the care team, and the genetic consultant agreed. Genetic testing was ordered for the causative genes, KMT2D and KDM6A, which detected a pathogenic variant in KMT2D: c. $15250 \mathrm{del}$, confirming the clinical diagnosis. This novel variant is predicted to result in a frameshift and premature protein termination: p.Leu5084Cysfs*6.

\section{"The cardinal signs of Kabuki syndrome (KS) make it possible to recognize in the newborn period, although it is often not diagnosed until later in infancy or childhood."}

\section{Discussion and counseling:}

The cardinal signs of Kabuki syndrome (KS) make it possible to recognize in the newborn period, although it is often not diagnosed until later in infancy or childhood. There are characteristic dysmorphic facial features with long palpebral fissures and lateral eversion of the lower lid. This appearance evoked the dramatic makeup used by traditional Kabuki actors, which is how the syndrome got its name. Eyebrows are arched and sparse laterally. The palate may be a cleft palate. Skeletal anomalies include brachydactyly,

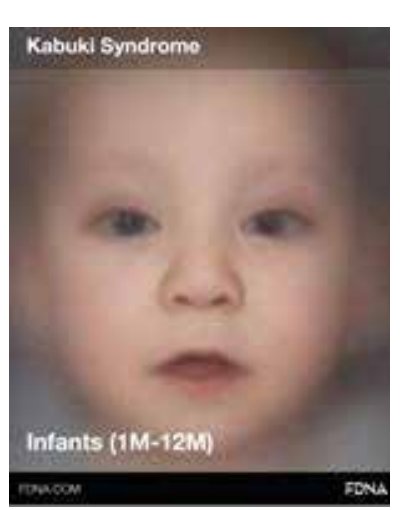
rib and vertebral anomalies, and hip dislocation. The prominent fetal pads persist in almost all affected infants. There is mild to moderate intellectual disability and postnatal growth deficiency. About $70 \%$ of patients with KS have a congenital heart defect, most commonly left-sided obstructive lesions.

The composite face in Figure 1 is a visualization of the KS phenotype in an infant that was generated by 
Face2Gene. This resource for phenotype matching is available at $h$ ttps://app.face2gene.com (and at the App store). This HIPAA compliant app can be used to upload and analyze patient photographs. Facial measurements and clinical features are used to match patient phenotypes with known genetic syndromes.

Transient hypoglycemia is common in all neonates. When it persists, it can be due to dysregulated insulin secretion by the pancreatic $\beta$-cells, with the high insulin level causing recurrent episodes of hypoglycemia. About $8-10 \%$ of patients with $\mathrm{KS}$ have neonatal or infantile hypoglycemia. Although hypoglycemia in response to hyperinsulinism $(\mathrm{HI})$ was considered rare, occurring in less than $1 \%$ of newborns with $\mathrm{KS}$, this is the second affected patient seen in our institution, which is why the cardiac care team queried the diagnosis of KS. In their review of 10 patients with Kabuki syndrome, Yap et al. noted that patients with $\mathrm{HI}$ do not necessarily present with high insulin levels, but they have inappropriate levels of insulin for their glucose measurements. Nine of the ten children with $\mathrm{KI}$ and hyperinsulinism reported by Yap responded to diazoxide therapy alone, but one did not, and that child required a partial pancreatectomy. These authors note that about $1 \%$ of neonates with $\mathrm{HI}$ have Kabuki syndrome. The underlying mechanism for the transient hyperinsulinism in KS is yet to be discerned. Growth hormone deficiency and adrenal insufficiency have been proposed as possible contributing factors.

Patients with Kabuki syndrome also have other endocrine perturbances, such as early breast development in girls, precocious puberty, growth hormone $(\mathrm{GH})$ deficiency, hypothyroidism, and diabetes insipidus; abnormal pituitary findings on magnetic resonance imaging have been rarely reported, with $\mathrm{GH}$ deficiency being the most common finding. Ongoing surveillance for endocrine dysfunction is recommended in patients in KS.

\section{Practical applications:}

1. Consider Kabuki syndrome in infants with long palpebral fissures and multiple congenital anomalies, especially those with left-sided cardiac lesions, with or without persistent hypoglycemia.

2. Investigate persistent hypoglycemia with insulin levels to document inappropriate or elevated insulin levels associated with hyperinsulinism.

3. Utilize phenotype-matching resources such as Face2Gene: https://app.face2gene.com

4. Confirm the clinical diagnosis of a genetic disorder with genetic testing whenever possible. This informs short-term management, long-term surveillance and ends the diagnostic odyssey for families and clinicians alike.
References:

1. Niikawa $N$, Kuroki Y, Kajii T, et al. Kabuki makeup (NiikawaKuroki) syndrome: a study of 62 patients. Am J Med Genet. 1988;31(3):565-589. PMID: 3067577

2. Yap KL, Johnson AEK, Fischer D, et al. Congenital hyperinsulinism as the presenting feature of Kabuki syndrome: clinical and molecular characterization of 9 affected individuals. Genet Med. 2019 Jan;21(1):233-242. Erratum in: Genet Med. 2018 Aug 11. PMID: 29907798.

3. https://app.face2gene.com

The authors have no relevant disclosures.

NT


Robin Clark, MD

Professor, Pediatrics

Loma Linda University School of Medicine

Division of Genetics

Department of Pediatrics

rclark@llu.edu



CONGENITAL CARDIOLOGY TODAY
The only worldwide monthly publication exclusively serving Pediatric and Adult Cardiologists that focus on Congenital/ Structural Heart Disease (CHD), and Cardiothoracic Surgeons.
Subscribe Electronically Free on the Home Page www.CongenitalCardiologyToday.com 